\title{
Mießner, Michael (2017): Staat - Raum - Ordnung: Zur raumordnungspolitischen Regulation regionaler Disparitäten
}

\author{
Münster - Westfälisches Dampfboot. = Raumproduktionen: Theorie und Gesellschaftliche \\ Praxis, Band 25. $356 \mathrm{~S}$.
}

\section{Simon Dudek ${ }^{1}$}

Eingegangen: 28. November 2017 / Angenommen: 15. Dezember 2017 / Online publiziert: 2. Januar 2018

๑) Springer-Verlag GmbH Deutschland, ein Teil von Springer Nature 2018

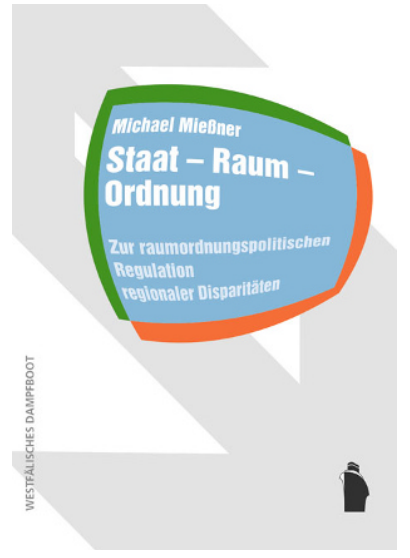

Eine prominente Rolle im Rahmen der Sondierungsgespräche im Herbst 2017 zur Bildung einer neuen Regierungskoalition nahmen Diskussionen über den Solidaritätszuschlag ein. Die kurz „Soli“ genannte Erhebung von Steuern zum Zwecke des Infrastrukturausbaus in den neuen Bundesländern dürfte das landläufig sinnfälligste Beispiel für den staatlichen Umgang mit regionalen Disparitäten sein. Die Unterstützung der Kommunen in der ehemaligen DDR folgt dabei einer Logik der Chancengleichheit, nach der der Staat äquivalente Ausgangsbedingungen in Ost und West herstellt. Zugleich, und dies entwickelte sich durch die Forschungsarbeiten von David Harvey, Neil Smith und anderen inzwischen zu sozialgeographischem Lehrbuchwissen, ist die ungleiche Entwicklung ein zentrales Charakteristikum kapitalistischer Vergesellschaftung.

Simon Dudek

simon.dudek@ku.de

1 Professur für Wirtschaftsgeographie, Katholische Universität Eichstätt-Ingolstadt, Ostenstraße 18, 85072 Eichstätt, Deutschland
In dieses Spannungsverhältnis aus raumordnungspolitischem Anspruch und ökonomischer Realität stößt die am Institut für Humangeographie der Goethe-Universität Frankfurt am Main eingereichte Dissertation von Michael Mießner, die 2017 unter dem Titel „Staat - Raum Ordnung“ als Band 25 der Reihe „Raumproduktionen“ im Verlag Westfälisches Dampfboot erschien. In dieser wendet er sich der Forschungsfrage $\mathrm{zu}$, wie die räumlich ungleiche Entwicklung in der Bundesrepublik Deutschland durch die Raumordnungspolitik prozessiert wird. Zur konkreten Fassung der Raumordnung greift der Autor dabei auf das Konzept des Staatsapparats zurück - in inhaltlicher Nähe zum marxistischen Staatstheoretiker Nicos Poulantzas, der damit der Vielschichtigkeit und Widersprüchlichkeit staatlicher Apparate innerhalb des Staates als Produkt gesellschaftlicher Auseinandersetzungen Ausdruck verleiht, und nicht zu Louis Althusser, der den Begriff im Zusammenhang mit der Reproduktion hegemonialer Ideologien verwendete.

Eben diese Staatsapparate, so eine der zentralen Argumentationslinien der vorliegenden Monographie, zeichnen sich durch eine hohe Kontinuität ordoliberaler Prinzipien aus. Als analytischen Bezugspunkt für seine Analyse wählt der Autor dabei den Grundsatz der „Gleichwertigkeit der Lebensverhältnisse“. Dieser harrt im raumordnungspolitischen Diskurs einer genaueren terminologischen Bestimmung, ist durchaus umkämpft und fungiert dementsprechend als ,hegemonialer leerer Signifikant“ (S. 44). Mit dieser konzeptionellen Anleihe in der poststrukturalistischen Theoriebildung schreibt Mießner den gleichwertigen Verhältnissen also das Potenzial zu, eine Vielzahl partikularer (auch potenziell konträrer) gesellschaftlicher Interessen zu vereinen. Zur Überprüfung dieser Hypothese greift der Autor auf eine breite Quellenbasis an raumordnungspolitischen Beiträgen und Wortmeldungen zurück. Das Gros der 
Grundlage seiner Analyse machen dabei Bundestagsreden und Gesetzestexte als legislative Zeugnisse politischer Auseinandersetzungen aus. Diese werden im Laufe der Analyse aber immer wieder durch die historisch entsprechenden Raumordnungsberichte des Bundesinstituts für Bau-, Stadtund Raumforschung (BBSR) bzw. seiner Vorgängerinstitute ergänzt.

Die Analyse bundesrepublikanischer Raumordnungspolitik nimmt demgemäß auch den Hauptteil in der Monographie ein. Nach einer Einleitung, in der der Autor die für seine Forschungsarbeit relevanten Begriffe und das Forschungsfeld kurz umreißt, dient Kapitel 2 dazu, den konzeptionellen und methodischen Rahmen der Arbeit darzustellen. Der politökonomischen Orientierung folgend führt Mießner darin regulationstheoretische Annahmen ein, um dem Nexus von Staat und Ökonomie gerecht zu werden. Diese dienen als Grundlage für die breiteren staatstheoretischen Überlegungen des Autors. Er übernimmt dabei Bob Jessops Arbeiten zum strategisch-relationalen Ansatz, orientiert sich aber weniger an dessen Luhmann-Exegese als vielmehr an seinen Arbeiten im Anschluss an Poulantzas. Vor allem dessen Staatsverständnis als ,materielle Verdichtung gesellschaftlicher Kräfteverhältnisse“ (Poulantzas 2002: 159) ermöglicht es, die ungleiche räumliche Entwicklung - verstanden als Dialektik von Angleichung und Differenzierung (vgl. Wissen/Naumann 2008) - als Konsequenz kapitalistischer Vergesellschaftung innerhalb des bürgerlichen Staates zu begreifen. Hier werden die Ähnlichkeiten zu Neil Brenners frühen Arbeiten zur deutschen Raumordnungspolitik und dem „state spatial process“ (Brenner 1997) deutlich. Mießner übernimmt diesen Begriff, der sich aus den gleichen konzeptionellen Wurzeln der Regulationstheorie und des strategisch-relationalen Staatsverständnisses ableitet. Zur Analyse dieser state spatial processes führt der Autor zum Ende des zweiten Kapitels die historischmaterialistische Politikanalyse ein, um ,die Verdichtung der Kräfteverhältnisse mittels Hegemonieprojekten nachzuvollziehen und zu analysieren" (S. 57). Diese umfasst in der vorliegenden Arbeit einerseits eine Kontextanalyse, in der - entsprechend der regulationstheoretischen Beschreibung von Krisen und Akkumulationsregimen - die breiteren gesellschaftlichen und ökonomischen Umstände in die Analyse einfließen. Andererseits bedient sich der Autor zur Akteur- und Prozessanalyse der Critical Discourse Analysis (vgl. unter anderem Fairclough 2013).

Die nachfolgenden Kapitel sind der Analyse der deutschen Raumordnungspolitik gewidmet. Analog zu Brenner differenziert der Autor dabei in Anlehnung an die Regulationsschule zwischen der Raumpolitik des Fordismus (Kapitel 3), der Krise des Fordismus (Kapitel 4), der Raumpolitik des Postfordismus (Kapitel 5) und der Raumordnungspolitik in der derzeitigen Finanz- und Wirtschaftskrise (Kapitel 6). Die Kapitel werden dabei getragen von der gegen- sätzlichen Auffassung der bundesrepublikanischen Raumordnungspolitik bei Brenner und Mießner. Erstgenannter spricht in der Phase des fordistischen Akkumulationsregimes von einem spatial keynesianism und erkennt in der Raumordnung des Postfordismus ein rescaled competition state regime. Dabei wird die Nähe zu Bob Jessops Arbeiten deutlich, der im Hinblick auf die sozialpolitische Entwicklung westlicher Industrienationen eine Entwicklung weg vom KWNS (keynesianian welfare national state) hin zum SWPR (schumpetrian workfare postnational regime) diagnostiziert (vgl. Jessop 1993). Dem widerspricht Mießner in seiner Arbeit. Anhand seiner Analyseergebnisse kann er nachvollziehbar darstellen, dass die Raumordnungspolitik in der Bundesrepublik durch eine hohe Kontinuität ordoliberaler Überzeugungen geprägt ist. So argumentiert der Autor (S. 310), dass Brenner in seinem Schluss auf eine keynesianische Raumordnungspolitik in der Phase des Fordismus „Zweck und Mittel“ staatlichen Handelns verwechsle. Vielmehr, so die vielleicht bedeutendste Schlussfolgerung der Arbeit, hat die „Raumordnungspolitik stets die real stattfindende räumliche Entwicklung unterstützt" (S. 307). So ist es auch zu erklären, dass der Grundsatz der gleichwertigen Lebensverhältnisse nicht für Ausgleichsbemühungen und eine Reduzierung von Disparitäten sorgte, sondern als leerer Signifikant fungierte, „hinter dem unterschiedliche parteipolitische Positionen verwischt wurden“ (S. 305).

Einen Erkenntnisgewinn bietet die vorliegende Dissertation in drei Punkten. Erstens fristet der staatstheoretische Zugang im Forschungskontext der Raumforschung noch ein Schattendasein. Dabei zeigt die vorliegende Analyse die Vorteile einer solchen Annäherung auf. So erklärt dieser Zugang die Persistenz von Überzeugungen im Staatsapparat und die Bedeutung leerer Signifikanten in der politischen Auseinandersetzung. Zweitens widerspricht die Arbeit der Annahme eines spatial keynesianism in allen westlich-industrialisierten Staaten und stellt dem das Konzept eines ordoliberalen deutschen Sonderwegs entgegen. Drittens wird den Lesern die Schwäche des Staatsapparats Raumordnung vor Augen geführt. Hierunter fällt die marginale Ausstattung des Staatsapparats mit Ressourcen, die begriffliche Unbestimmtheit zentraler Grundsätze und die mangelnde Gestaltungskraft zur Eindämmung regionaler Disparitäten.

Die Regionalforschung setzte im Kontext der Konjunktur endogener Regionalentwicklung den Fokus stark auf die Analyse von Bottom-up-Prozessen. Michael Mießners Buch „Staat - Raum - Ordnung“ kann in diesem Zusammenhang durchaus als antizyklisches Forschungsprodukt aufgefasst werden. Gerade in Zeiten, in denen eine Renaissance des Nationalstaats diskutiert wird und die räumlichen Disparitäten unter den Stichworten der Peripherisierung und der sterbenden Dörfer an gesellschaftlicher Relevanz gewinnen, lohnt es aber, die Top-down-Strukturen bundesrepublikanischer Raumordnungspolitik verstärkt in 
den Blick zu nehmen, um bestehende Ungleichheiten erklären zu können. Dies ist das Verdienst der besprochenen Arbeit.

\section{Literatur}

Brenner, N. (1997): State territorial restructuring and the production of spatial scale: Urban and regional planning in the Federal Republic of Germany, 1960-1990. In: Political Geography 16, 4, 273-306. https://doi.org/10.1016/S0962-6298(96)00003-0
Fairclough, N. (2013): Critical discourse analysis: The critical study of language. Abingdon.

Jessop, B. (1993): Towards a Schumpeterian workfare state? Preliminary remarks on post-Fordist political economy. In Studies in Political Economy 40, 1, 7-39. https://doi.org/10.1080/19187033. 1993.11675409

Poulantzas, N. (2002): Staatstheorie: politischer Überbau, Ideologie, autoritärer Etatismus. Hamburg.

Wissen, M.; Naumann, M. (2008): Die Dialektik von räumlicher Angleichung und Differenzierung: Zum uneven-developmentKonzept in der radical geography. In: ACME - An International E-Journal for Critical Geographies 7, 3, 377-406. 